\title{
The ATLAS b-jet Trigger
}

\author{
Danilo Enoque FERREIRA DE LIMA** \\ University of Glasgow \\ E-mail: dferreir@mail.cern.ch
}

The ATLAS detector, at the LHC, has a three-level trigger, which selects events relevant for the physics goals of the experiment. The identification of jets arising from bottom quark production is important in many analyses. $b$-tagging relies on the fragmentation of the $b$ quark, which generates a $B$ hadron, that retains most of the parent quark's momentum $(\sim 70 \%)$. Furthermore, the high $b$ quark mass results in decay products with high momenta with respect to the jet axis. The lifetime tagger relies on the the relatively long lifetime of the $B$ hadrons ( $\sim 1.6 \mathrm{ps}$ in their rest frame), which allows them to have a long decay length. Due to the large mass of the $B$ hadron, the tracks reconstructed from this decay often have large impact parameters, compared to prompt jets. The algorithm exploits this by identifying tracks from the $B$ hadron decay which are displaced from the primary interaction vertex and thus, indicate that a long-lived particle was present. The latest performance results with 2011 data are shown.

XXIst International Europhysics Conference on High Energy Physics

21-27 July 2011

Grenoble, Rhône-Alpes France

\footnotetext{
* Speaker.

†n behalf of the ATLAS Collaboration.
} 


\section{Introduction}

The experiments at the Large Hadron Collider (LHC) are currently taking data with $\sqrt{s}=$ $7 \mathrm{TeV}$. The ATLAS detector [1] is a general purpose detector with a tracking system, a calorimetric system and a muon spectrometer further away from the beam pipe. The three-tiered ATLAS Trigger has been developed to cope with the high event rate and the low cross section of the processes of interest. The Level 1 (L1) is implemented in hardware and uses the calorimeters and muon spectrometer with coarse granularity to select Regions of Interest (RoI), which will be analysed by the following levels. The Level 2 (L2) trigger and subsequent Event Filter (EF) are software based and use, in addition, the tracking system and the full granularity of the detectors.

The ATLAS b-jet Trigger uses a calorimeter-based jet trigger at L1. At L2 and EF, it uses the tracking information to implement the same algorithm in both levels, with more refined estimates of the track parameters at the EF. Due to the high rate of events, each level has a limitation in the processing time. In particular, the L2 has $\sim 40 \mathrm{~ms}$ to reject or accept an RoI, and EF has $\sim 4 \mathrm{~s}$, allowing more complex algorithms.

\section{2. b-jet Trigger Selection}

The lifetime taggers calculate the significance of the transverse $\left(d_{0}\right)$ and longitudinal $\left(z_{0}\right)$ impact parameters of each track in a jet cone, assigning a sign to each track. $d_{0}$ is the distance of closest approach of the track to the $z$ axis, and $z_{0}$ is the $z$ coordinate of the point of closest approach to the origin. The significances are given by: $S\left(z_{0}\right)=\left(z_{0}-z_{p v}\right) / \sigma\left(z_{0}\right)$ and $S\left(d_{0}\right)=d_{0} / \sigma\left(d_{0}\right)$. The sign of the $S\left(z_{0}\right)$ or $S\left(d_{0}\right)$ is positive if the track intersects the jet direction in the $y-z$ plane or transverse plane projection, respectively. The jet axis direction is calculated through an average of the pseudo-rapidity and azimuthal angle of the tracks.

The JetProb tagger is the default tagger and calculates the probability that each track comes from the primary vertex (PV), using a fitted resolution function $\mathscr{R}(x)$. The track probability is $\mathscr{P}_{\text {track }}=\int_{-\infty}^{-\left|S\left(d_{0}\right)\right|} \mathscr{R}(x) d x$. This probability is then, combined in a single probability for the whole jet, which is expected to be close to zero for $b$-jets. This method is robust because the resolution function is fitted in data, and not Monte Carlo.

The selected tracks are required to have a minimum transverse momentum of $1 \mathrm{GeV} / \mathrm{c}$, a maximum $d_{0}$ of $1 \mathrm{~mm}$ and $z_{0}-z_{p v}$ not exceeding $2 \mathrm{~mm}$. The tracks must also have a good fit quality and match the RoI. The PV is approximated as being at the origin of the $x-y$ plane. The $z$ coordinate of the PV is calculated by histogramming the $z_{0}$ of all selected tracks in the RoI and using a sliding window algorithm to select the largest local maximum.

A second class of triggers has been developed to collect a sample enriched in $b$-jets without explictly using $b$-tagging algorithms, which can be used for commissioning. Such triggers require a geometrical matching between a muon and a jet, since the branching ratio of $B$ hadrons decaying in muons is $\sim 20 \%$.

\section{Data/MC comparison and Offline bias}

The comparisons of the signed transverse impact parameter, the transverse momentum of the tracks and the number of selected tracks in the RoI at the Event Filter are shown in Figures 1, 2, 3. 
It can be seen that there is good agreement between data and Pythia-generated QCD Monte Carlo (MC) simulations. Figure 4 shows the distribution of the offline JetProb tagger in data and a Pythiagenerated MC simulation of QCD events. The $-\log _{10}$ (offline JetProb) is histogrammed, which has high values for $b$-jets. The red, blue and green points show the distribution after different cuts at the trigger JetProb. It can be seen that jets tagged with the $b$-jet trigger have an enhanced fraction of heavy flavour, using the Offline tagger as a reference. The only scaling in this figure is applied to Monte Carlo, which is normalised to the unbiased data points.



Figure 1: Data/MC comparison of the significance of the signed transverse impact parameter.

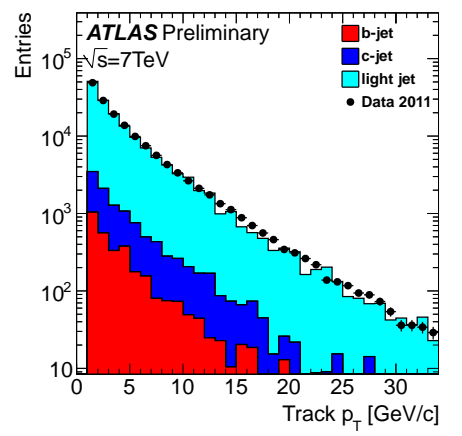

Figure 2: Data/MC comparison of Figure 3: Data/MC comparison the transverse momenta of tracks at the Event Filter.

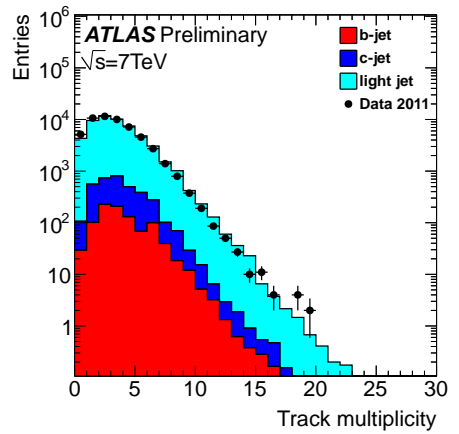

of the multiplicity of tracks at the Event Filter.

\section{Outlook}

Starting from the beginning of 2011, the ATLAS $b$-jet trigger has been active and selecting events enriched in heavy flavour. Datadriven measurements of the online and offline $b$ tagging efficiencies and mis-tagging rate are currently being finalised. Several ATLAS physics analyses are currently based on $b$-jet triggers, such as: top-antitop analyses with fully hadronic top quark decays, searches for supersymmetric extensions with 0 leptons in the final state and searches for Higgs decaying in bottomantibottom quark pairs. One example of a $b$ jet trigger, which is used for the Higgs decaying in bottom-antibottom searches, requires two

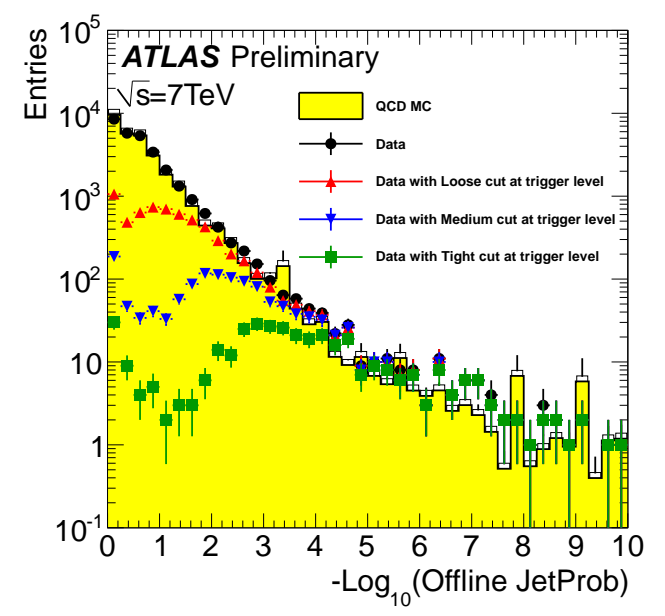

Figure 4: Effect of the $b$-tagging in the Offline tagging variable. medium trigger $b$-tagged jets and at least four $\mathrm{L} 1$ jets, with a minimum energy cut of $10 \mathrm{GeV}$ at $\mathrm{L} 1$ per jet. New taggers are under study to be used by the beginning of 2012, including a likelihood ratio tagger and a secondary vertex-based tagger.

\section{References}

[1] G. Aad et al. [ The ATLAS Collaboration ], [arXiv:0901.0512 [hep-ex]]. 